\title{
Contaminación del aire en la Florida (Talca, Chile): Beneficios económicos en salud por la reducción de los niveles $\mathrm{PM}_{10}$
}

\author{
ARCADIO A. CERDA ${ }^{\mathrm{a}}$, LEIDY Y. GARCÍA ${ }^{\mathrm{b}}$
}

\section{Hypothetical health and economic benefits associated with a reduction in air pollution in a Chilean city (Talca)}

Background: Environmental air pollution is associated with a higher incidence of respiratory diseases, absenteeism and costs. Aim: To model the health related economic benefits associated to a reduction in air pollution and the resulting lower prevalence of respiratory diseases, in a Chilean city. Material and Methods: A time series model for year 2006 was elaborated. The dependent variable was the number of consultations for respiratory disease. The independent variables were air pollution expressed as particulate matter of 10 micrometers or less $\left(P M_{10}\right)$, minimum and maximum environmental temperatures, environmental humidity and number of consultations for chronic diseases. Results: The variables that best explained the number of consultations for respiratory diseases were $P M_{10}$, minimal environmental temperatures and preexisting respiratory diseases. In a hypothetical scenery of a $67 \%$ reduction in $P M_{10}$, 69\% of medical consultations for respiratory diseases would be avoided. This would result in a net saving of US\$345,000 per year. Conclusions: The reduction in $P M_{10}$ emissions would result in an important reduction in consultations for respiratory diseases and monetary savings.

(Rev Med Chile 2010; 138: 1395-1402).

Key words: Air pollution; Public health; Respiratory tract diseases.

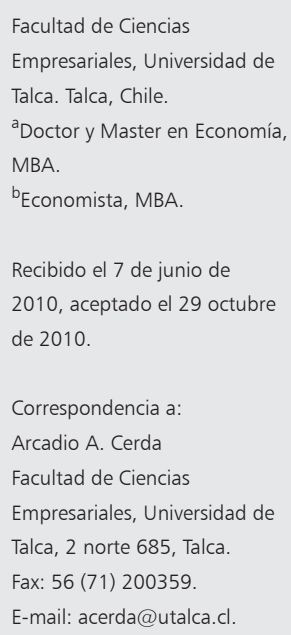

L os impactos de la contaminación del aire en la salud de las personas se ha estudiado extensivamente, en especial en áreas metropolitanas, donde los impactos varían dependiendo de los contaminantes y sus fuentes, donde las emisiones son generadas principalmente por el tráfico vehicular y las actividades productivas ${ }^{1-6}$.

La evidencia muestra que las personas de más escasos recursos están más expuestas a problemas ambientales generando problemas de equidad ${ }^{1,7}$. Igualmente, la contaminación generada en el hogar es preocupante, de acuerdo a la Organización Mundial de la Salud ${ }^{8}$, pues el humo proveniente de combustibles sólidos quemados en el hogar es unos de los principales riesgos para las personas que viven en países en vía de desarrollo. El uso de dichos combustibles contribuye a altas tasas de enfermedades respiratorias agudas y crónicas ${ }^{8,10}$. Dichas emisiones también genera impactos ambientales a las poblaciones aledañas, usuarios y no usuarios de combustibles sólidos, que incluso están dispuestos a pagar por medidas de control ambiental o certificación de leña ${ }^{11}$.

Autores como Ségala et $\mathrm{al}^{6}$ encuentran que las consultas médicas (CM) de emergencia a hospitales por bronquitis se relacionan con la contaminación del aire en invierno, donde el análisis de series de tiempo muestra asociaciones entre 
contaminación del aire, mortalidad, enfermedades cardiorrespiratorias y admisiones a centros de salud. Dicho estudio muestra una correlación negativa entre emisiones con temperatura, lluvia y viento. Algunos estudios también incluyen la humedad ${ }^{12,13}$.

Adicionalmente, numerosos estudios han determinado, en diversas urbes, incluida la ciudad de Santiago de Chile ${ }^{14}$, que la contaminación con $\mathrm{PM}_{10}$ determina un aumento de mortalidad por enfermedades cardiovasculares y broncopulmonares.

En Chile, el mejoramiento en la calidad del aire en las principales ciudades del país ha sido una prioridad gubernamental por años. Se han realizado diagnósticos e inventarios de emisiones en las principales ciudades del país, lo que ha permitido iniciar las gestiones para reducir la contaminación generada por la combustión de leña y la actividad industrial ${ }^{1}$. Sin embargo, en ciudades fuera del área metropolitana, sólo algunas ciudades han realizados estudios y tomados medidas concretas con respecto a la contaminación del aire ${ }^{15,16}$.

En la ciudad de Talca, un informe de la Secretaria Regional Ministerial de Salud ${ }^{17}$ indica que la ciudad superó 44 veces la norma que establece un máximo de $\mathrm{PM}_{10}$ de $150 \mu \mathrm{g} / \mathrm{m}^{3}{ }^{18}$, alcanzando un máximo de 327 y $283 \mu \mathrm{g} / \mathrm{m}^{3}$, para los años 2004 y 2005, respectivamente, mientras que el año 2006 se superó 21 veces la norma (Tabla 1 ).

La contaminación atmosférica se produce principalmente en invierno, por las bajas temperaturas que aumenta el uso de combustibles sólidos,

Tabla 1. Límites referenciales de $\mathrm{PM}_{10}$ por categoría

\begin{tabular}{|cc|}
\hline Categoría PM10 & $\begin{array}{c}\text { Límites promedio día } \\
\boldsymbol{\mu g} / \mathbf{m} \mathbf{3}\end{array}$ \\
\hline Bueno & $000-149$ \\
\hline Regular & $150-194$ \\
\hline Mala & $195-239$ \\
Crítico & $240-329$ \\
\hline Peligroso & $>320$ \\
\hline
\end{tabular}

Decreto Supremo 59 establece norma contaminante de Material Particulado Respirable $\mathrm{PM}_{10}$ en ciento cincuenta microgramos por metro cúbico normal $\left(\mu \mathrm{g} / \mathrm{m}^{3}\right)$ como concentración de 24 horas. Categorías sólo referenciales. principalmente leña, para calefaccionar los hogares, causando daños en la salud de las personas, principalmente enfermedades respiratorias, lo que aumenta las consultas diarias, hospitalizaciones y tasas de mortalidad ${ }^{19}$. Por ello, es importante determinar el impacto que tiene la contaminación del aire sobre la salud de los habitantes.

El objetivo de este estudio es estimar los beneficios económicos en salud que generaría un menor número de enfermedades respiratorias de la población vía una reducción en los niveles de $\mathrm{PM}_{10}$ respirable en uno de los sectores con mayor contaminación del aire en la ciudad de Talca, Chile.

La hipótesis es que la reducción del contaminante $\mathrm{PM}_{10}$ reduciría significativamente las consultas médicas (CM) por enfermedades respiratorias y a su vez los costos económicos asociados.

\section{Materiales y Métodos}

\section{Marco conceptual}

El aire es un bien público que requiere métodos alternativos para determinar valor que le dan los individuos. Un método de valoración monetaria es asignar valor a una función de dosis-respuesta ${ }^{20}$.

La ecuación (1) relaciona la calidad del aire en función del nivel de emisiones ${ }^{21,22}$ :

(1) $C=C(e), \frac{d C}{d e}<0$

donde $C=$ calidad del aire y $e=$ nivel de emisiones del contaminante. La ecuación (2) relaciona la calidad del aire, las acciones de tipo defensiva y el efecto que estas generan en la salud de las personas, donde existe una relación inversa:

(2) $D=D(C, Y(C)), \frac{\partial D}{\partial C}<0, \frac{\partial D}{\partial Y(C)}<0$

con $D=$ índice de efectos en la salud del individuo; $Y(C)=$ acción defensiva en salud del individuo, en función de la calidad del aire. El valor monetario depende de los índices de salud de los individuos, donde existe una relación positiva entre ambas variables (ecuación 3).

(3) $V=V(D), \frac{d V}{d D}>0$

donde $\mathrm{V}=$ valor monetario del estado de salud de los individuos. Sustituyéndose, entre ecuaciones 
(1), (2) y (3) se obtiene:

$$
\text { (4) } V=V\{C(e), Y(C)\}
$$

Freeman ${ }^{21}$ considera que los beneficios de una política de reducción de niveles de emisiones de $e^{0}$ a $e^{1}$ implica una mejora en la calidad del aire de $C^{0}$ a $C^{1}$, con el consecuente impacto en los beneficios:

(5) $B=\Delta V=V\{C(e), Y(C)\}^{1}-V\{C(e), Y(C)\}^{0}$

A pesar de la ventaja del modelo para explicar los beneficios, tiene limitaciones porque requiere valorizar la reducción de emisiones mediante la implementación de políticas ambientales, lo que se aleja del objetivo del estudio. Por ello, se consideró una adaptación del modelo, según lo planteado por Sánchez et $\mathrm{al}^{5}$.

El modelo primero estima el impacto de la contaminación atmosférica en las CM, a través de la estimación de la elasticidad, la que mide el cambio porcentual en las CM con respecto a un cambio porcentual en los niveles de $\mathrm{PM}_{10}$. Segundo, se valora económicamente los beneficios de reducir la contaminación vía el ahorro de costos por morbilidad (ecuación 6):

(6) $\Delta B=B(\Delta X)$

donde $\Delta B=$ beneficios por reducción en casos de enfermedades respiratorias y $\Delta X=$ variación de casos de morbilidad por cambios en la calidad ambiental.

Azqueta $^{23}$ menciona tres enfoques para valorar los efectos en la morbilidad ante cambios en la calidad ambiental: costo de tratamiento por enfermedad, funciones de producción de salud y valoración contingente. En este trabajo se aplicó el primer método ${ }^{24,25}$, porque el método de funciones de producción de salud posee problemas con la determinación del gasto médico óptimo y valoración contingente requiere entrevistas personales $^{26}$.

Este estudio tiene similitud a otros realizados previamente ${ }^{27,28}$, sin embargo, se diferencia en la modelación del costo de la calidad ambiental vía $\mathrm{CM}$ y no diferencia entre grupos etarios, porque el tamaño de la muestra era insuficiente.

\section{Especificación del modelo}

Basado en la literatura, se estimó un modelo (función Dosis-Respuesta) de series de tiempo en primeras diferencias y con rezagos, cuya variable dependiente fueron las CM por enfermedades respiratorias y las independientes el nivel $\mathrm{PM}_{10}$, la temperatura mínima, promedio y máxima, la humedad y las consultas por enfermedades crónicas. El modelo es estimado usando el procedimiento de mínimos cuadrados ordinarios (MCO).

El modelo se expresa como ecuación (7):

(7) $C M_{t}=C M_{t}\left(C M_{t-\delta_{c m}}, P M 10_{t-\delta_{P M 10}}, T_{t-\delta_{T}}, H_{t-\delta_{H}}, C R_{t-\delta c_{R}}\right)+\varepsilon_{t}$

donde $C M_{t}=\mathrm{CM}$ por enfermedades respiratorias momento t, $P M 10_{t}$, = nivel $\mathrm{PM}_{10}$ en día $\mathrm{t} ; T_{t}=$ temperatura en momento $\mathrm{t} ; H_{t}=$ humedad momento t; $C R_{t}=$ enfermedad respiratoria crónicas momento t; y $\varepsilon_{t}=$ error aleatorio. La serie temporal disponible de emisiones es día por medio, donde los rezagos de las respectivas variables $(\delta)$ hay que multiplicarlos por dos para llevarlo a número de días.

El análisis se delimitó en dos aspectos: considera sólo enfermedades relacionadas con la contaminación atmosférica y la población de referencia fueron las personas atendidas en el consultorio de la Florida, Talca, reportados enfermos durante el año 2006. Dada la limitante de información existente, no se han agregado los accidentes cardiovasculares que se ha demostrado que aumentan como consecuencia de la contaminación con material particulado fino ${ }^{27}$, y tampoco se ha valorado el aumento de la mortalidad por exposición previa a material particulado ${ }^{14}$. Además, la exposición crónica a dicho material aumenta la mortalidad por diversos cánceres, en especial, el broncopulmonar $^{28}$.

Un detalle de las CM se muestra en Tabla 2, diferenciadas en agudas y crónicas (CR). Se incluyen las CR en el modelo para aislar en efecto de estas consultas con las que están directamente relacionadas con el $\mathrm{PM}_{10}$.

\section{Área de estudio y datos}

El estudio se realizó en el sector la Florida, Talca. La elección de esta ciudad se debe principalmente por el riesgo que poseía de ser declarada zona de saturación por contaminantes atmosféricos y a la falta de estudios relacionados. Además, dicho sector exhibe altos índices de contaminación por combustión a leña.

Los datos de las CM por enfermedades respi- 
Tabla 2. Estadística consultas médicas respiratorias por tipo de enfermedad, 2006

\begin{tabular}{|lrlr|}
\hline Enfermedades agudas & \multicolumn{3}{l|}{ Enfermedades crónicas } \\
\hline Bronquitis/Sinusitis/ Laringitis & 1.681 & Asma & 500 \\
Resfrió común & 1.401 & Bronquitis/ sinusitis/laringitis crónica & 32 \\
Neumonía & 697 & Rinofaringitis y faringitis crónica & 11 \\
Faringitis & 469 & Otras pulmonares crónicas & 856 \\
Influenza & 175 & & 1.399 \\
Amigdalitis & 138 & & \\
Otras & 48 & & \\
Total & 4.609 & & \\
\hline
\end{tabular}

Fuente: Consultorio la Florida, Talca.

Tabla 3. Resumen estadístico de valores promedio de niveles de $\mathrm{PM}_{10}$, temperatura y temperaturas máximas y mínimas

\begin{tabular}{|lcccc|}
\hline & Media & Máxima & Mínima & Desviación estándar \\
\hline PM $_{10}\left(\mathrm{ug} / \mathrm{m}^{3}\right)$ & 60,53 & 301 & 0 & 52,07 \\
TMAX $\left(\mathrm{C}^{\circ}\right)$ & 21,71 & 35 & 6 & 6,91 \\
TMIN $\left(\mathrm{C}^{\circ}\right)$ & 7,99 & 16 & 0 & 3,60 \\
TPROM $\left(\mathrm{C}^{\circ}\right)$ & 14,83 & 24 & 3 & 4,80 \\
\hline
\end{tabular}

Fuente: Elaborado en base a información del Centro de Investigación y Transferencia en Riego y Agroclimatolgía de la Universidad de Talca.

ratorias provienen del Consultorio de Salud de la Florida, único centro que se encuentra en el sector del estudio. Esto podría generar un sesgo porque las personas pueden ir al hospital por una consulta.

Los datos de material particulado, temperatura y humedad, fueron suministrados por el Centro de Investigación y Transferencia en Riego y Agroclimatología de la Universidad de Talca.

\section{Resultados}

El promedio de CM por enfermedades respiratorias diarias disponibles fue de 33 consultas, éstas corresponden a todos los días del año excluyendo domingos y festivos, con un total de $6.008 \mathrm{CM}$ el 2006. Las enfermedades respiratorias agudas más frecuentes consultadas en el año fueron bronquitis $(24,23 \%)$, resfrío común $(23,32 \%)$, y neumonitis $(11,6 \%)$. La causa más usual de CM por enfermedades respiratorias crónicas en el consultorio fueron enfermedades pulmonares obstructivas crónicas (14,25\%) (Tabla 2).
La Tabla 3 muestra la estadística descriptiva de la variables bajo análisis. Las correlaciones de las variables objeto de estudio muestran una relación negativa entre $\mathrm{PM}_{10} \mathrm{y}$ temperatura, lo inverso sucede entre $\mathrm{PM}_{10}$, CM y CR (Tabla 4).

Un aumento del $\mathrm{PM}_{10}$ en $1 \%$, incrementará las $\mathrm{CM}$ y $\mathrm{CR}$ en $34 \%$ y $89 \%$, respectivamente. La correlación $C M_{t}$ con $P M 10_{t-\delta P M 10}$ con $\delta_{P M 10}=$ 1 a 13 se presenta en Tabla 5, reflejando una alta correlación por varios días después de la emisión.

La relación existente entre $\mathrm{PM}_{10}$ y CM se ilustra en la Figura 1, a mayor nivel de $\mathrm{PM}_{10}$ respirable las consultas por enfermedades respiratorias se ven incrementadas, observándose la presencia de rezagos en la relación.

\section{Modelo econométrico}

La ecuación (8) muestra el modelo econométrico final estimado:

(8) $\ln C M_{t}=\alpha \ln P M_{t-4}+\beta \ln C M_{t-7}+\delta T M I N_{t-2}+\gamma C R_{t-3}+\varepsilon_{t}$ El modelo explica $54,1 \%$ de la variabilidad de 
Beneficios económicos en reducción de contaminación del aire - A. Cerda et al

Tabla 4. Matriz de correlaciones de niveles de $\mathrm{PM}_{10}$, temperatura, humedad relativa y consultas por enfermedades respiratorias la Florida, Talca, 2006

\begin{tabular}{|c|c|c|c|c|c|c|}
\hline & CM & PM10 & TMIN & TMAX & TPROM & CR \\
\hline $\mathrm{CM}$ & 1 & & & & & \\
\hline $\mathrm{PM}_{10}$ & $+0,34$ & 1 & & & & \\
\hline TMIN & $-0,28$ & $-0,51$ & 1 & & & \\
\hline TMAX & $-0,27$ & $-0,45$ & $+0,60$ & 1 & & \\
\hline TPROM & $-0,30$ & $-0,53$ & $+0,81$ & 0,95 & 1 & \\
\hline$C R$ & $+0,89$ & $+0,28$ & $-0,25$ & $-0,19$ & $-0,23$ & 1 \\
\hline
\end{tabular}

$\mathrm{CM}=$ consultas médicas por enfermedades respiratorias. $\mathrm{CR}=$ consultas médicas por enfermedades respiratorias crónicas.

Tabla 5. Correlaciones $\mathrm{CM}$ versus $\mathrm{PM}_{10}$

\begin{tabular}{|ccc|}
\hline $\begin{array}{c}\text { Rezagos } \mathbf{P M}_{10} \\
\left(\delta_{\mathbf{P M} 10}\right)\end{array}$ & Días & Correlación \\
0 & 0 & 0,34 \\
1 & 2 & 0,45 \\
\hline 2 & 4 & 0,30 \\
\hline 3 & 6 & 0,29 \\
\hline 4 & 8 & 0,40 \\
\hline 5 & 10 & 0,33 \\
\hline 6 & 12 & 0,23 \\
\hline 7 & 14 & 0,30 \\
\hline 8 & 16 & 0,36 \\
\hline 9 & 18 & 0,25 \\
\hline 10 & 20 & 0,32 \\
\hline 11 & 22 & 0,32 \\
\hline 12 & 24 & 0,24 \\
\hline 13 & 26 & 0,10 \\
\hline
\end{tabular}

Un rezago equivale a dos días.

las consultas médicas. Como se evidencia en la Tabla 6, los coeficientes de las variables son significativamente diferentes de cero a un nivel de significación de 5\%, con excepción de la variable humedad que se eliminó. Además, no hay autocorrelación de primer orden y no se puede rechazar la hipótesis de homosedasticidad.

Las estimaciones muestran que las CM están dependiendo del nivel del

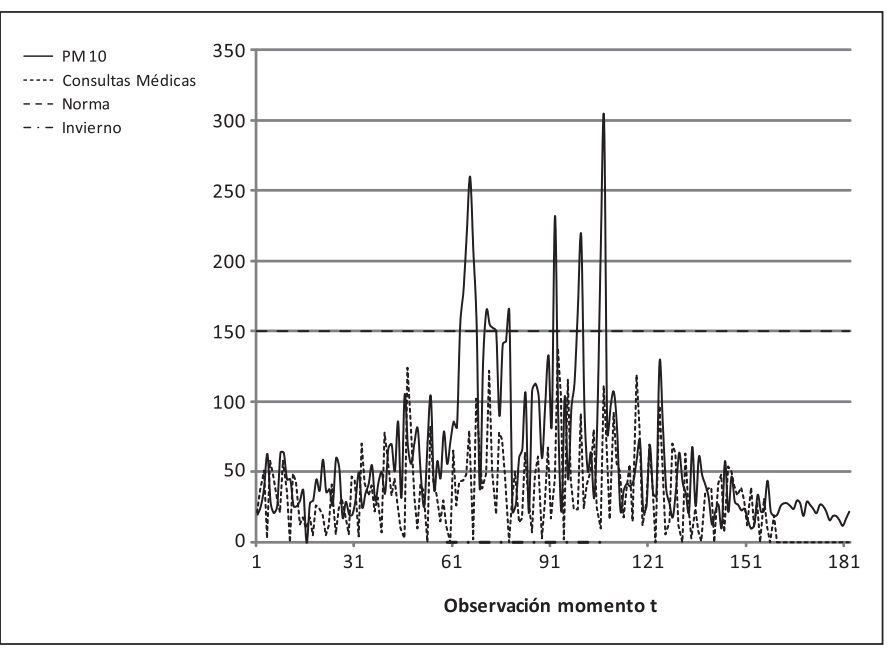

Figura 1. Relación $\mathrm{PM}_{10}$ y consultas médicas respiratorias (CM). Fuente: Elaboración de los autores con base en información del Centro de Investigación y Transferencia en Riego y Agroclimatología de la Universidad de Talca con base en datos de la CONAMA.

Tabla 6. Resultados modelo econométrico

\begin{tabular}{|ccc|}
\hline Variable & Coeficiente & t-estadístico \\
$\mathrm{PM}_{\mathrm{t}-4}$ & $0,469^{1}$ & 3,13 \\
$\mathrm{CM}_{\mathrm{t}-\mathrm{7}}$ & $0,547^{1}$ & 7,92 \\
\hline $\mathrm{TMIN}_{\mathrm{t}-2}$ & $-0,570^{1}$ & $-2,15$ \\
$\mathrm{CR}_{\mathrm{t}-3}$ & $0,217^{1}$ & 3,74 \\
\hline
\end{tabular}

$R^{2}=0,514 ; \mathrm{F}$ estadístico $=17,92 ; \mathrm{n}=177$ observaciones. $\mathrm{H}$ de Durbin $=$ 0,106 ; test de White $=1,78$. 'valor-p $<0,04$ del estadístico t-student. Lo subíndices de las variables reflejan el número de rezagos de la variable. Dado que los datos disponibles son cada dos días, cada rezago hay que multiplicarlo por dos para llevarlo a número de días. 
material particulado, la temperatura mínima, las CM previas, y las CR con ocho, cuatro, catorce y seis días previos, respectivamente. La temporalidad de las variables se debe a que la serie contiene datos de día por medio. Además, el impacto del $\mathrm{PM}_{10}$ se refleja en que la correlación cruzada con las CM alcanza un valor de $+0,34$.

Se calculó la elasticidad del cambio porcentual en CM ante un cambio porcentual en los niveles de $\mathrm{PM}_{10}$ igual a 0,469 . Al suponer que en los registros aparece un efecto contagio en los individuo ${ }^{29}$, es necesario dividir la elasticidad por uno menos el coeficiente de rezago de la variable endógena $(0,547)$ obteniéndose la elasticidad de largo plazo ajustada de 1,0375 .

\section{Mejora en la calidad del aire}

En este apartado se estiman los beneficios de reducir la contaminación del aire. Se comenzó con el cálculo del número de consultas evitadas que se obtienen del mejoramiento de la calidad del aire, tomando el promedio de $\mathrm{PM}_{10}$ del período muestral alcanzando $60,53 \mu \mathrm{g} / \mathrm{m}^{3}$, y contando el número de días del período en que se supera la norma promedio diaria de latencia de $120 \mu \mathrm{g} / \mathrm{m}^{3}$ estipulado en la Ley de Bases de Medio Ambiente. La norma de latencia se sobrepasa cuando las emisiones llegan a $80 \%$ del valor establecido en la norma diaria de calidad del aire, equivalente a un límite máximo de la norma $\left(150 \mu \mathrm{g} / \mathrm{m}^{3}\right)$, lo que ocurrió en 21 días. Adicionalmente, el PM10 no puede exceder más de 7 veces al año la norma, para ser considerada la calidad del aire como tolerable. El cálculo realizado fue:

(20) $60,53 / 21=2,88=X / 7$ de donde $X=20,16$

A partir de ecuación (20) resulta:

(21) $\{(60,53-20,16) / 60,53\}^{\star} 100=66,69 \%$

De manera que las concentraciones del $\mathrm{PM}_{10}$ se reducen en $66,69 \%$ de su nivel promedio.

\section{Consultas evitadas}

El análisis de las consultas evitadas requirió tener los datos del número de consultas por enfermedades respiratorias en el sistema público de salud del sector de estudio. A dichas consultas se les aplicó la elasticidad promedio con respecto al $\mathrm{PM}_{10}$, calculada con base a las elasticidades de largo plazo. A partir de esto se obtuvo que con una reducción de $66,60 \%$ en las concentraciones de $\mathrm{PM}_{10}$ se reducirían las CM en $69,10 \%$ $\left(1,0375^{\star} 0,6669^{\star} 100\right)$, equivalente a una disminución de 4.157 en las consultas.

\section{Ahorro de costos por consultas evitadas}

Los costos asociados por enfermedad respiratoria, son costos directos, costos indirectos (incluye el costo de oportunidad del tiempo perdido) y disminución en bienestar de los individuos por las molestias ${ }^{29}$. Los dos primeros costos se estiman con la información disponible, pero el tercero no se considera porque requiere valoración de los afectados.

El costo directo considerado es el valor de la consulta médica. Con datos de las principales enfermedades respiratorias del Departamento de Salud de Talca, se calculó el costo promedio de consulta por enfermedad respiratoria en $\$ 27.750$.

Los costos indirectos relacionados con la menor productividad laboral o costo de oportunidad de dejar de trabajar por enfermedad o cuidar a un enfermo, se cuantifico usando el salario promedio diario de una persona. Los costos por día de ausentismo laboral se utilizó el salario promedio mensual imponible en la Región del Maule de \$288.878, según la Encuesta de Caracterización Socioeconómica (CASEN) de $2006^{30}$; y se consideró que hay 21,7 días laborales al mes para doce meses laboralmente. El costo del ausentismo laboral diario es $\$ 13.312$. Si el costo de la actividad restringida equivalente al $20 \%$ del día laboral y la reducción de la productividad laboral es un tercio del $80 \%$ del trabajo realizado, el costo total por actividad restringida diaria es $\$ 6.212$. El costo indirecto promedio considero la información referente a los días de reposo promedio por patologías respiratorias y los niveles etarios de los afectados, estimándose un valor de $\$ 4.421$.

\section{Discusión}

Los resultados obtenidos con el modelo, en términos de coeficiente de determinación, superan los alcanzados por otros a nivel regional (Fuentes P, Benavides P. Beneficios económicos por una reducción de contaminación atmosféri- 
ca para el sector la Florida, ciudad de Talca, año 2006. Memoria de Grado para optar al título de Ingeniero Comercial, Universidad de Talca, 2007. Carrasco C, Richard C. Beneficios económicos de contaminación atmosférica según grupos etarios en Temuco y Padre Las Casas. Memoria de Grado para obtener el título de Ingeniero Comercial, Universidad de Concepción, año 2003). Similar a otros estudio ${ }^{6,12,13}$, las variables que mejor explican las CM respiratorias son el material particulado, temperatura mínima y enfermedades respiratorias preexistentes, confirmando los resultados de múltiples estudios epidemiológicos, apoyados con investigaciones de metaanálisis, que muestran una asociación entre contaminación por partículas e incremento en pacientes con enfermedades respiratorias y muertes ${ }^{19}$, a pesar que algunos estudios muestran una baja o inexistente relación entre $\mathrm{PM}_{10}$ y síntomas respiratorios ${ }^{31,32}$.

Bajo el escenario hipotético de cumplimiento de la norma de latencia, implicaría un ahorro de 133,7 millones de pesos anuales, valor inferior al obtenido por Cerda et $\mathrm{al}^{11}$ utilizando el método de valoración contingente. Dichos resultados se pueden comparar con los costos que significan la implementación de medidas tendiente a mitigar el daño ambiental, vía un organismo certificador de leña seca o tecnologías sustitutas.

Cabe mencionar que las estimaciones de este estudio dan un valor mínimo de referencia del costo real total de la contaminación, el que se espera sea mayor al valor estimado, porque sólo se ha considerado en su cálculo el efecto sobre el incremento de enfermedades respiratorias agudas, pero no se ha considerado el aumento de morbilidad y mortalidad aguda por enfermedades cardiovasculares ni la morbilidad y mortalidad por cáncer broncopulmonar causada por exposición crónica a material $\mathrm{PM}_{10}{ }^{14,27,28}$.

Por lo anterior, políticas públicas destinadas a reducir los niveles de emisión generados por la combustión a leña en la ciudad generaría disminuciones en las $\mathrm{CM}$ y beneficios económicos importantes para la sociedad.

Agradecimientos: Se agradece la colaboración de las autoridades del Consultorio de la Florida, Talca por permitir tener acceso a la información utilizada en este estudio y a la compilación realizada de dicha información por las Ingeniero Comercial Pía A. Fuentes y Paula A. Benavides.

\section{Referencias}

1. Chun-Quan O, Hedleya A, Chunga R, Thacha T, Chaua $\mathrm{Y}$, Chana K, et al. Socioeconomic disparities in air pollution-associated mortality. Environmental Research 2008; 107: 237-44.

2. Analitis A, Katsouyanni K, Dimakopoulou K, Samoli E, Nikoloulopoulos K, Petasakis Y, et al. Short-term effects of ambient particles on cardiovascular and respiratory mortality. Epidemiology 2006; 17: 230-3.

3. Katsouyanni K, Touloumi G, Samoli E, Gryparis A, Le TA, Monopolis Y, et al. Confounding and effect modification in the short-term effects of ambient particles on total mortality: results from 29 European cities within the APHEA2 project. Epidemiology 2001; 12: 521-31.

4. Samoli E, Touloumi G, Schwartz J, Anderson HR, Schindler C, Forsberg B, et al. Short-term effects of carbon monoxide on mortality: an analysis within the APHEA project. Environ Health Perspect 2007; 115, 1578-83.

5. Sánchez J, Valdés S, Ostro B. Estimación de los beneficios en salud del plan de descontaminación de Santiago. El Trimestre Económico 1998; 65: 363-406.

6. Ségala C, Poizeau D, Mesbah M, Willems S, Maidenberg M. Winter air pollution and infant bronchiolitis. Environmental Research 2008; 106: 96-100.

7. O’Neill MS, Jerrett M, Kawachi I, Levy JI, Cohen AJ, Gouveia N, et al. Health, wealth, and air pollution: advancing theory and methods. Environ Health Perspect 2003; 111: 1861-70.

8. World Health Organization. The World Health Report 2002. Disponible en: http://www.who.int/whr/2002/ en/2002 [Consultado el 20 Agosto 2009].

9. Zhao BX, Wang S, Aunan K, Seip HM, Hao J. Air pollution and lung cancer risks in China -a meta-analysis. Sci Total Environ 2006; 366: 500-13.

10. Staff $\mathrm{H}$, Aunan K, Seip H. Potential health benefit of reducing household solid fuel use in Shanxi province, China. Science of the Total Environment 2006; 372: 120-32.

11. Cerda AA, García LY, Bahamondez A, Poblete V. Comparación de la disposición a pagar por políticas de mejoramiento de la calidad del aire entre usuarios y no usuarios de calefacción de combustión a leña (Talca, Chile). Lecturas de Economía 2010; 72: 195-212.

12. Zeka A, Zanobetti A, Schwartz J. Short term effects of particulate matter on cause specific mortality: effects of lags and modification by city characteristics. Occup Environ Med 2005; 62, 718-25.

13. Aga E, Samoli E, Touloumi G, Anderson HR, Cadum E, Forsberg B, et al. Short-term effects of ambient particles on mortality in the elderly: results from 28 cities in the APHEA2 project. Eur Respir J Suppl 2003; 40: 28-33. 
Beneficios económicos en reducción de contaminación del aire - A. Cerda et al

14. Ostro B, Sánchez JM, Aranda C, Eskerland GS. Air pollution and mortality: results from a study of Santiago, Chile. J Exposure Anal Environ Epidemiol 1996; 6: 97114.

15. CONAMA (Comisión Nacional del Medio Ambiente). Priorización de Medidas de Reducción de Emisiones por Uso Residencial de Leña para la Gestión de la Calidad del Aire en Temuco y Padre Las Casas. Universidad de Concepción 12 de diciembre de 2002. Disponible en: http://www.sinia.cl/1292/articles-28474_recurso_1.pdf [Consultado el 13 agosto 2009].

16. CONAMA (Comisión Nacional del Medio Ambiente). Descontaminación Atmosférica en Centros Urbanos. Disponible en: http://www.conama.cl/portal/1301/propertyvalue-15492.html 2006. [Consultado el 10 marzo 2009].

17. Secretaria Regional Ministerial de Salud, Región del Maule. Informe Técnico de Monitoreo Calidad del Aire año 204, 2005 y 2006. Julio 31, 2007.

18. Decreto Supremo 59 (D.S. 59). Establece norma primaria de calidad del aire para material particulado respirable MP10, en especial los valores que definen situación de emergencia. Secretaría General de la Presidencia. Diario Oficial, junio 25, 1998.

19. Zamorano A, Márquez S, Aránguiz J, Bedregal P, Sánchez I. Relación entre bronquitis aguda con factores climáticos y contaminación ambiental. Rev Med Chile 2003; 131: 1117-22.

20. Pearce D. Economía Ambiental. Editorial Longman Group Limited, Londres. 1985. p. 43-7.

21. Freeman IIIM. The measurement of environmental and resource values, theory and methods. Washington, D.C.: Resources for the Future; 1993.

22. Freeman IIIM. Air and Water Pollution Control. 2da edición, Limusa, México; 1995.

23. Azqueta D. Valoración Económica de Calidad Ambiental. 1ra edición, Mc Graw Hill, Madrid; 1994.

24. Vásquez F, Cerda A, Orrego S. Valoración económica del ambiente. Thomson Learning. Buenos Aires, Argentina; 2007.

25. Cerda A, Rojas J, García L. Disposición a pagar por un mejoramiento en la calidad ambiental en el Gran Santiago, Chile. Lecturas de Economía 2007; 67: 143-60.

26. Field B. Economía Ambiental. Editorial Mc Graw-Hill, Colombia, 1995.

27. Brook RD, Brook JR, Urch B, Vincent R, Rajagopalan S, Silverman F. Inhalation of fine particulate air pollution and ozone causes acute arterial vasoconstriction in healthy adults. Circulation 2002; 105: 1534-36.

28. Pope CA $3^{\text {rd }}$, Burnett RT, Thun MJ, Calle EE, Krewski $\mathrm{D}$, Ito $\mathrm{K}$, et al. Lung cancer, cardiopulmonary mortality, and long term exposure to fine particulate air pollution. JAMA 2002; 287: 1132-41.

29. Sánchez J, Morel T. Una Estimación de los Beneficios en Salud de Reducir la Contaminación Atmosférica en Santiago. En: Economía del Medio Ambiente en América Latina, Juan I. Varas Editor, Ediciones Universidad Católica de Chile; Diciembre de 1995.

30. CASEN. Encuesta de Caracterización Socioeconómica. Disponible en: http://www.mideplan.cl/casen/index. html [Consultado el 10 marzo 2009].

31. Roemer W, Hoek G, Brunekreef B, Clench-AAS J, Forsberg B, Pekkanen J, et al. PM10 elemental composition and acute respiratory health effects in European children (PEACE project). Eur Respir J 2000; 15: 553-9.

32. Osunsanya T. Prescott G, Seaton A. Acute respiratory effects particles: mass or number Occup Environ Med 2001; 58: 154-9. 